\title{
Recubrimientos protectores de TiN y AIN: comportamiento frente a temperatura
}

\author{
M.A. AUGER ${ }^{1,2}$, O. SÁNCHEZ ${ }^{1}$ Y J.M. ALBELLA ${ }^{1}$ \\ 'Instituto de Ciencia de Materiales de Madrid (ICMM-CSIC) 28049 Cantoblanco. Madrid (España). \\ ${ }^{2}$ Centro Nacional de Investigaciones Metalúrgicas (CENIM-CSIC). Avda. Gregorio del Amo, 8. 28040 Madrid (España).
}

\begin{abstract}
La utilización de materiales cerámicos como el nitruro de titanio o de aluminio ha experimentado una gran popularización en los últimos años gracias a su gran variedad de aplicaciones. Una importante aplicación que cuenta hasta el momento con resultados muy favorables es la de su uso como recubrimientos protectores de herramientas industriales, así como de moldes empleados en la fabricación de diferentes elementos. Una de las técnicas más utilizadas en la obtención de recubrimientos protectores es la técnica de sputtering, utilizada en este trabajo. En el presente estudio se han analizado las propiedades estructurales y mecánicas de láminas delgadas de TiN y AlN mediante las técnicas de difracción de rayos X, microscopía electrónica de barrido, nanoindentación y pin-on-disk. Dichas capas han sido sometidas a un tratamiento térmico en el que se han aplicado tres temperaturas: $750^{\circ} \mathrm{C}, 850^{\circ} \mathrm{C}$ y $950^{\circ} \mathrm{C}$, y se han observado variaciones en sus propiedades antes y después del tratamiento. Las capas de TiN presentan una resistencia térmica en torno a $750^{\circ} \mathrm{C}$. Las capas de AlN resisten hasta una temperatura en torno a $850^{\circ} \mathrm{C}$, observándose además una mejoría en sus propiedades al ser sometidas a dicha temperatura de $850^{\circ} \mathrm{C}$.
\end{abstract}

Palabras clave: TiN, AlN, sputtering reactivo, recubrimientos protectores, resistencia térmica

\section{Protective TiN and AIN coatings: behaviour after temperature treatments.}

The use of ceramic materials like titanium and aluminium nitrides have experienced a great improvement in the last years due to the variety of applications they show. The use as protective coatings on industrial tools is one important application with very favorable results for the moment, as it is the use as coating molds used for different elements fabrication. One of the mostly often used techniques to deposit protective coatings is the sputtering technique, which has been used in this work. In the present study we have analysed structural and mechanical properties of TiN and AlN thin films, using X-Ray diffraction, scanning electron microscopy, nanoindentation and pin-on-disk techniques. Thermal treatments have been applied on the mentioned films, applying three different temperature values: $750^{\circ} \mathrm{C}, 850^{\circ} \mathrm{C}$ y $950^{\circ} \mathrm{C}$. Changes in their properties before and after the treatments have been observed. TiN layers show a thermal resistance around $750^{\circ} \mathrm{C}$. The thermal resistance for AlN layers is around $850^{\circ} \mathrm{C}$, being also observed an improvement in their properties when applying $850^{\circ} \mathrm{C}$ of thermal treatment.

Keywords:TiN, AlN, reactive sputtering, protective coatings, thermal resistance

\section{INTRODUCCIÓN}

El uso de nitruros metálicos en forma de lámina delgada se ha extendido en los últimos años a diferentes áreas científicas y tecnológicas debido a la gran variedad de aplicaciones que ofrecen: como recubrimientos protectores, decorativos, barreras de difusión en circuitos integrados (1-3), etc. Uno de los nitruros metálicos que mayor popularidad ha alcanzado es el nitruro de titanio, cuyo uso, principalmente como recubrimiento protector, ha sido extensamente implantado y aplicado con fines industriales (4), obteniéndose un excelente comportamiento debido a sus excepcionales propiedades de dureza, adherencia al substrato, estabilidad química y termodinámica, etc. Concretamente, en un trabajo previo realizado en nuestro laboratorio, se aplicaron recubrimientos de TiN sobre cuchillas de acero inoxidable utilizadas en el corte de fibra de vidrio y se obtuvo como resultado un aumento del $67 \%$ en el tiempo de vida de la herramienta (5). El hecho de retardar el reemplazo de piezas posee un beneficio económico, al retrasar las paradas de producción, y medioambiental, al reducir la producción de residuos. Otro nitruro metálico, también muy utilizado, sobre todo en aplicaciones ópticas y electrónicas, es el nitruro de aluminio (6). Pero además de sus buenas propiedades eléctricas, el nitruro de aluminio posee una gran estabilidad química y elevada dureza, lo cual permite su uso como recubrimiento protector (7), que será el aspecto analizado para este material.

En el presente trabajo se han depositado capas de TiN y AlN en forma de lámina delgada mediante la técnica de sputtering magnetrón reactivo. Dichas capas han sido sometidas a tratamientos térmicos a diferentes temperaturas: 750,850 y $950^{\circ} \mathrm{C}$, y se han analizado sus características mecánicas y estructurales antes y después del tratamiento. Es importante analizar el comportamiento de estos materiales frente a la temperatura puesto que, para poder ser utilizados satisfactoriamente como recubrimientos protectores en procesos industriales se requiere una estabilidad térmica de los mismos, ya que en dichos procesos es típico alcanzar temperaturas elevadas. 


\section{PARTE EXPERIMENTAL}

Las capas de TiN y AlN analizadas en este trabajo, de 600 y $450 \mathrm{~nm}$ de espesor respectivamente, han sido depositadas mediante la técnica de sputtering magnetrón reactivo según los parámetros experimentales descritos en un trabajo anterior (8). Los substratos utilizados han sido obleas de silicio monocristalino de tipo p con orientación (100).

Una vez depositadas las capas y fuera de la cámara de sputtering se han realizado tratamientos térmicos a presión atmosférica aplicando tres temperaturas: 750,850 y $950^{\circ} \mathrm{C}$, con el fin de observar el comportamiento de las capas ante temperaturas típicamente alcanzables en procesos industriales. Las muestras han sido sometidas a tratamientos de $60 \mathrm{~min}$ para cada una de las temperaturas mencionadas.

Las técnicas de caracterización utilizadas en este trabajo han sido: difracción de rayos $\mathrm{X}$, microscopía electrónica de barrido, nanoindentación y pin-on-disk. Los diagramas de difracción de rayos $X$ se han obtenido en un equipo Siemens D-5000, operando en modo $\theta-2 \theta$. El microscopio electrónico utilizado ha sido un equipo JEOL6500F con filamento de emisión de campo. Las medidas de dureza se han realizado en un nanoindentador Micro Materials Nanotest 300, aplicando cargas en un rango entre 0.5 y $200 \mathrm{mN}$ sobre una punta tipo Berkovitch, y en el análisis de los resultados se ha seguido el método de Oliver y Pharr (9). Los ensayos de pin-on-disk se han llevado a cabo en un tribómetro Microtest TPD/10. En ellos se ha utilizado una bola de WC-Co de $3 \mathrm{~mm}$ de diámetro girando sobre la superficie de las muestras a 300rpm. Se ha aplicado una carga de $1 \mathrm{~N}$, el diámetro de la trayectoria ha sido de $4 \mathrm{~mm}$ y la longitud recorrida de $12.5 \mathrm{~m}=1000 \mathrm{rev}$.

\section{RESULTADOS Y DISCUSIÓN}

\subsection{Caracterización de TiN sometido a tratamiento térmico}

En la figura 1 se muestran los difractogramas obtenidos, en el modo $\theta-2 \theta$, para el recubrimiento de TiN original y

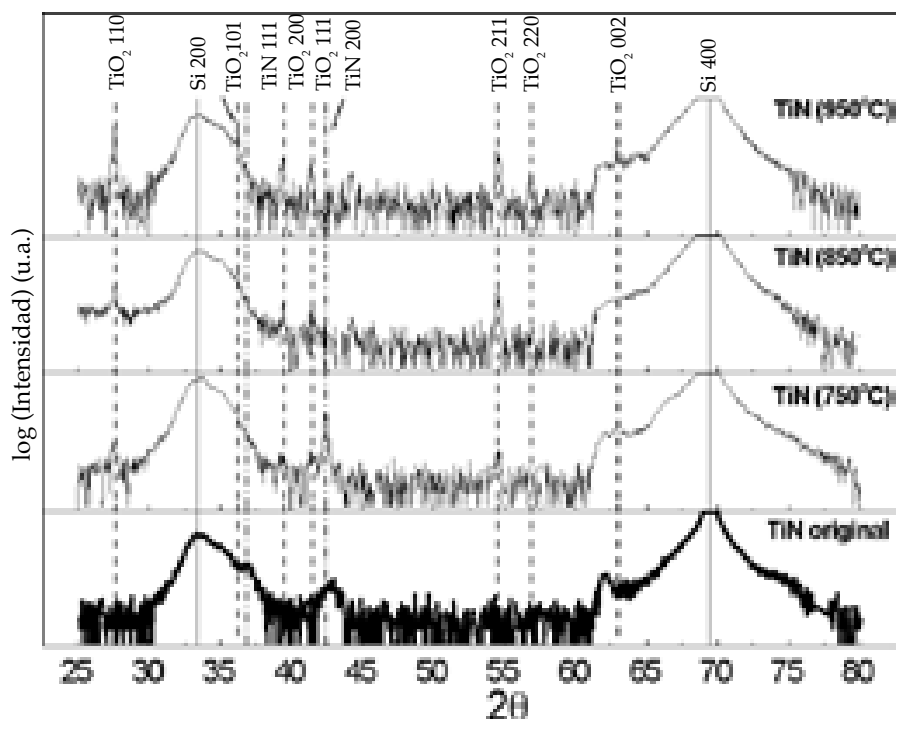

Fig. 1.- Difractogramas de rayos $X$ para la muestra de TiN original $y$ sometida a los tratamientos térmicos. tras ser sometido a cada uno de los tratamientos térmicos mencionados. Se puede observar que el recubrimiento original presenta una estructura cúbica fcc (10). En el diagrama obtenido tras el tratamiento térmico a $750^{\circ} \mathrm{C}$ durante $1 \mathrm{~h}$ la muestra aún presenta la estructura cúbica fcc, aunque se puede apreciar cómo empiezan a aparecer difracciones de planos asociados al $\mathrm{TiO}_{2}$ tetragonal (10). En el diagrama obtenido tras el tratamiento a $850^{\circ} \mathrm{C}$ la intensidad de los picos asociados a la estructura de TiN cúbico presenta una intensidad muy baja, mientras que la de los picos asociados al $\mathrm{TiO}_{2}$ tetragonal es mayor que en el diagrama anterior, apareciendo además nuevas difracciones. En el difractograma obtenido tras el tratamiento térmico a $950^{\circ} \mathrm{C}$ prácticamente se anula la estructura cúbica de TiN y predomina la formación de $\mathrm{TiO}_{2}$.

La conclusión que se desprende tras este análisis es que el recubrimiento de TiN es estable al menos hasta una temperatura de $750^{\circ} \mathrm{C}$, hasta la cual mantiene su estructura cúbica. Tras el tratamiento térmico a temperaturas superiores se observan importantes variaciones en la capa: aparece un importante deterioro en la estructura cúbica del material y, según los resultados obtenidos, también en su composición, ya que la estructura observada tras el tratamiento térmico revela la formación de $\mathrm{TiO}_{2}$ en fase tetragonal.

En la figura 2 se muestra la morfología superficial de la capa de TiN original y tras ser sometida a los tratamientos térmicos. En ella se pueden observar variaciones en el tamaño de grano de las partículas, que sufren un engrosamiento a medida que aumenta la temperatura de tratamiento. Dicho engrosamiento coincide con la incorporación de oxígeno en la capa, que se detecta en los difractogramas de rayos $X$ mostrados en la Fig. 1 y en un análisis adicional por EDX.

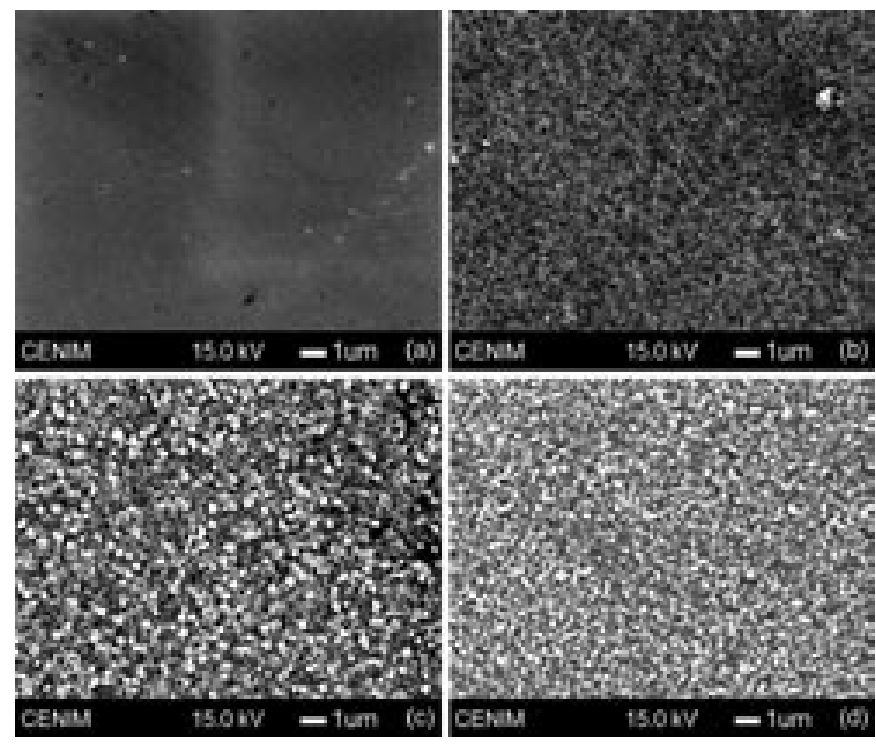

Fig. 2.- Imágenes SEM de las muestras a) $\mathrm{TiN}$ original, b) $\mathrm{TiN}\left(750^{\circ} \mathrm{C}\right)$, c) $\operatorname{TiN}\left(850^{\circ} \mathrm{C}\right)$ y d) $\mathrm{TiN}\left(950^{\circ} \mathrm{C}\right)$.

La dureza de esta serie de muestras ha sido analizada mediante la técnica de nanoindentación, aplicando diferentes cargas entre 0.5 y $200 \mathrm{mN}$ y obteniendo valores de dureza a diferentes profundidades en el perfil de la capa. En la figura 3-a) se representan gráficamente los valores de dureza en función del cociente $\mathrm{d} / \mathrm{h}$ (eje horizontal inferior), donde $\mathrm{d}$ es la profundidad a la cual se ha realizado la medida $\mathrm{y} h$ es el 

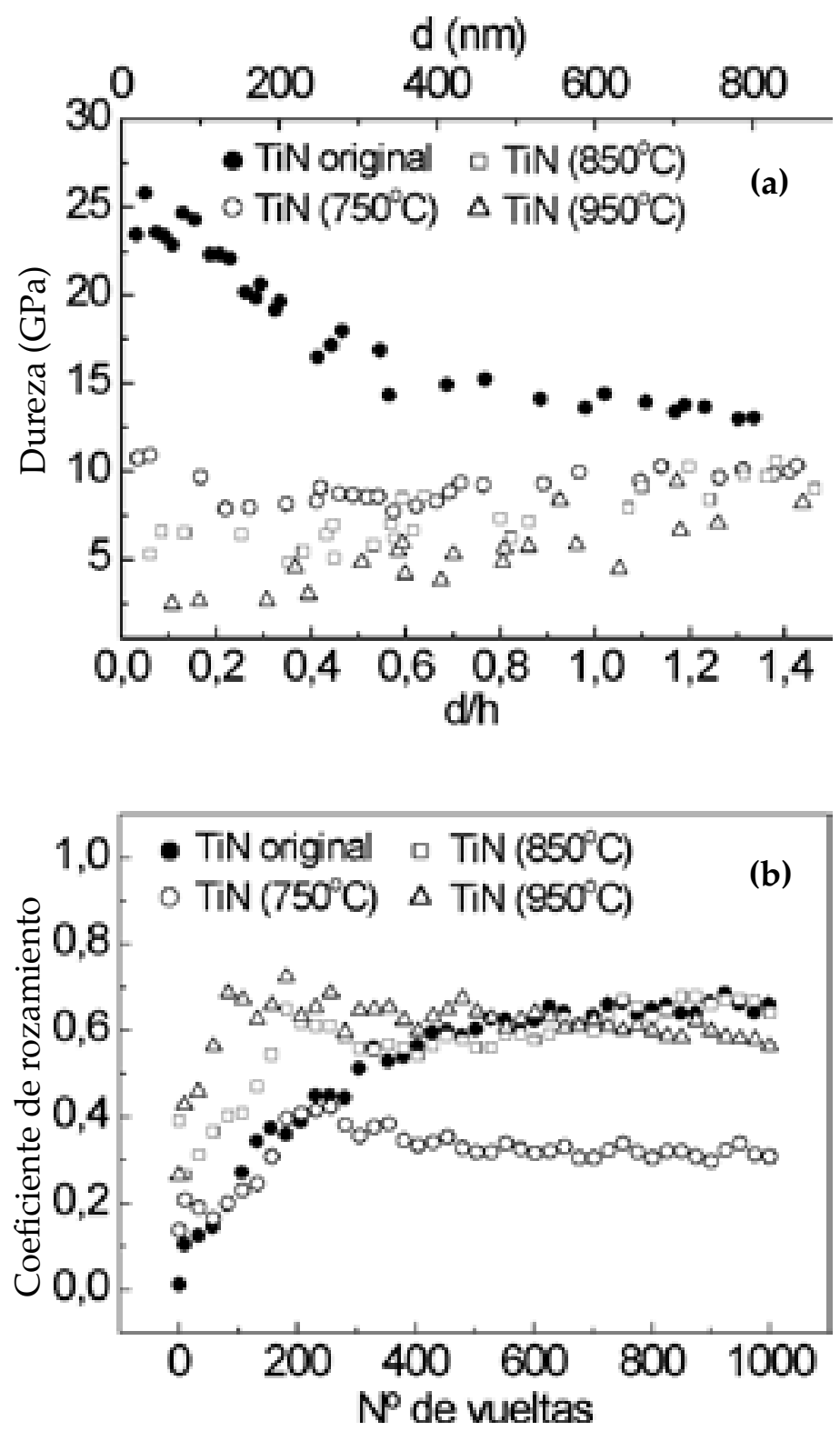

Fig. 3.-a) Perfiles de dureza y b) coeficiente de rozamiento vs. $\mathrm{N}^{o}$ de vueltas del ensayo para la muestra de TiN original y sometida a los tratamientos térmicos.

espesor de la capa (600nm). En el eje horizontal superior de la figura 3-a) se indica, en $\mathrm{nm}$, la profundidad a la que se ha realizado la indentación. Los valores que se muestran en la Tabla I corresponden al promedio estadístico realizado dentro del $10 \%$ del espesor total de la capa $(\mathrm{d} / \mathrm{h}<0.1)$, para así evitar posibles influencias del substrato en la medida (11). En dicha tabla observamos un importante descenso en la dureza de la capa tras el tratamiento térmico a $750^{\circ} \mathrm{C}$. Este comportamiento está relacionado con el deterioro estructural y morfológico observado en el análisis de rayos X y en la imagen SEM de la figura 2-b). Del mismo modo tras los tratamientos realizados a 850 y $950^{\circ}$ se observa un deterioro progresivo de la capa, cuya dureza termina estando por debajo de la del substrato de silicio (10GPa). En estos dos últimos casos se observa además una mayor dispersión en los valores de las medidas, debida principalmente a inhomogeneidades generadas en la superficie de la película tras el proceso de oxidación, como se puede observar en las figuras 2-c) y d).

En la figura 3-b) se representa cómo varía el coeficiente de rozamiento de las capas en función del número de vueltas del ensayo de pin-on-disk, y en ella se puede observar que la capa sometida a $750^{\circ} \mathrm{C}$ presenta un comportamiento similar a la del recubrimiento original en las primeras vueltas del ensayo, mientras que las capas sometidas a temperaturas superiores presentan valores más altos de dicho coeficiente desde el inicio del ensayo.

Los resultados obtenidos en este estudio muestran, por tanto, un comportamiento estable del recubrimiento de TiN, tanto estructural como mecánico, para temperaturas de hasta $750^{\circ} \mathrm{C}$.

\subsection{Caracterización de AlN sometido a tratamiento térmico}

En la figura 4 se muestran los difractogramas obtenidos, en el modo $\theta-2 \theta$, para el recubrimiento de AlN original y tras ser sometido a cada uno de los tratamientos térmicos. Se observa que el recubrimiento original presenta una estructura hexagonal (10). En los diagramas obtenidos tras el tratamiento térmico a 750 y $850^{\circ} \mathrm{C}$, la muestra aún presenta dicha estructura, en cambio, en el diagrama obtenido después del tratamiento térmico a $950^{\circ} \mathrm{C}$ se observa una degradación en la estructura al descender notablemente la intensidad de los picos detectados en los diagramas anteriores. Según este análisis, el recubrimiento de AlN permanece estable hasta una temperatura de al menos $850^{\circ} \mathrm{C}$. Tras el tratamiento térmico a una temperatura superior se observan variaciones en la estructura de la capa, perdiéndose la definición de la fase hexagonal. En cambio, mediante el análisis de rayos $\mathrm{X}$ realizado no se detectan cambios en la composición, como una posible formación de óxido de aluminio, ya que no se registran picos de difracción asociados a la presencia de dicho compuesto en forma cristalina, pudiendo estar presente en forma amorfa, la cual no es detectable mediante rayos $\mathrm{X}$.

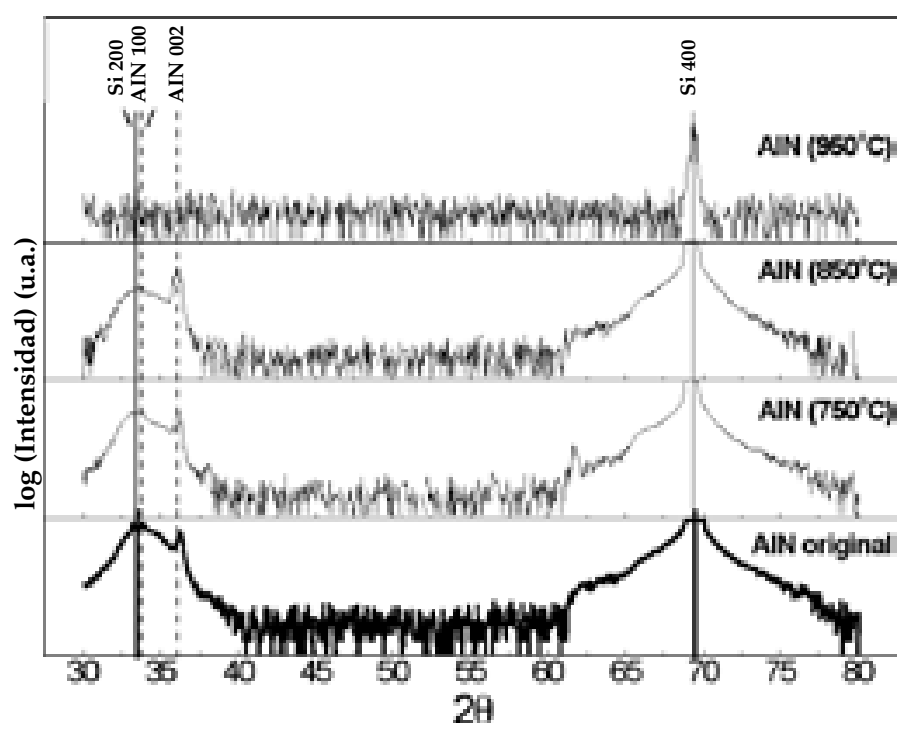

Fig. 4.- Difractogramas de rayos $\mathrm{X}$ para la muestra de AlN original $\mathrm{y}$ sometida a los tratamientos térmicos. 

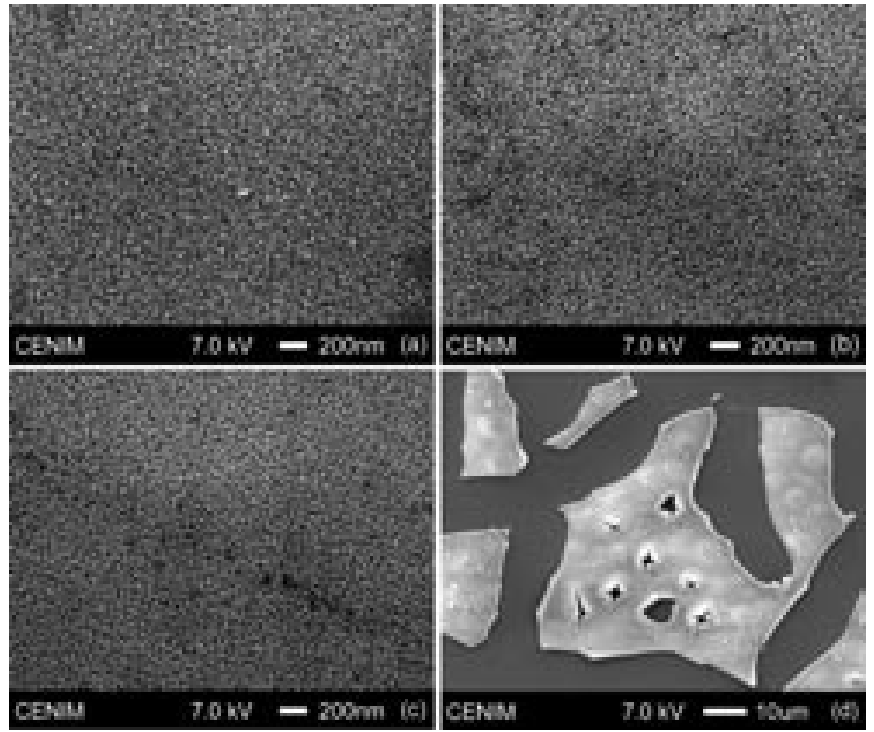

Fig. 5.- Imágenes SEM de las muestras a) AlN original, b) $\mathrm{AlN}\left(750^{\circ} \mathrm{C}\right)$, c) $\operatorname{AlN}\left(850^{\circ} \mathrm{C}\right)$ y d) $\operatorname{AlN}\left(950^{\circ} \mathrm{C}\right)$.
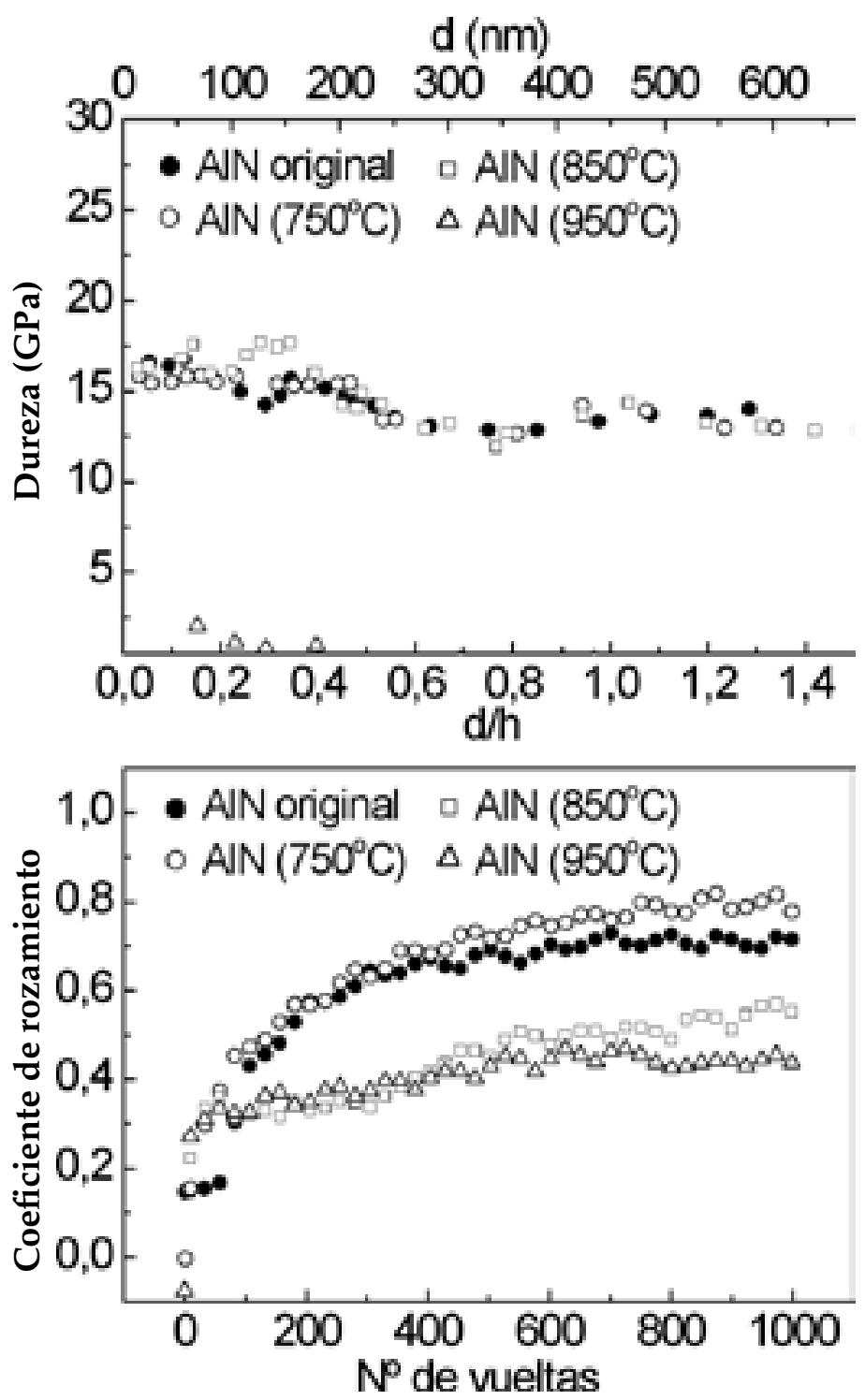

Fig. 6.- a) Perfiles de dureza y b) coeficiente de rozamiento vs. $N^{o}$ de vueltas del ensayo para la muestra de AlN original y sometida a los tratamientos térmicos.
En la figura 5 se muestran imágenes SEM de la superficie de las muestras analizadas. En ellas se puede observar que el tamaño de grano de las partículas es similar para la muestra de AlN original y sometida a $750^{\circ} \mathrm{C}$. Para la muestra sometida a $850^{\circ} \mathrm{C}$ se detecta una disminución del tamaño de grano, lo cual coincide con el estrechamiento del pico 002 observado en la figura 4. En la muestra sometida a $950^{\circ} \mathrm{C}$ se detecta por EDX una incorporación de oxígeno en la capa $(31 \%)$ que genera grietas por aumento de volumen y da lugar, por tanto, a la formación de islas, como se puede ver en la figura 5-d).

En la figura 6-a) se presentan los perfiles de dureza de la serie de muestras obtenidos mediante nanoindentación. $\mathrm{Al}$ igual que en la figura 3-a) se representan los valores de dureza en función de $\mathrm{d}$ y del cociente $d / h$. En dicha figura podemos observar un comportamiento de la dureza similar para la muestra original y para la muestra que ha sido sometida a tratamiento térmico a $750^{\circ} \mathrm{C}$. En cambio, tras realizar el tratamiento a $850^{\circ} \mathrm{C}$ podemos observar que se produce un endurecimiento de la capa, apareciendo en este caso una dureza superior a la del recubrimiento original. Dicho endurecimiento coincide con la reducción del tamaño de grano observado en la Fig. 5-c). Para la muestra sometida a $950^{\circ} \mathrm{C}$ se obtienen valores muy bajos de dureza, relacionados con el deterioro estructural de la capa observado en el análisis previo por rayos $\mathrm{X}$ y con su morfología superficial. Los valores de dureza obtenidos para cada una de las capas analizadas se muestran en la Tabla I.

En la figura 6-b) se muestran los valores del coeficiente de rozamiento en función del número de vueltas de ensayo, y en ella se puede ver que la capa de $\mathrm{AIN}$ sometida a $850^{\circ} \mathrm{C}$ presenta un coeficiente de rozamiento más bajo que la muestra original sin producirse la fractura de la capa. En la muestra sometida a $950^{\circ} \mathrm{C}$ el coeficiente de rozamiento es también menor que el de la muestra original, pero en este caso ya hemos observado un deterioro importante en la capa mediante el resto de técnicas utilizadas.

Se confirma entonces tras la realización de este estudio, que las capas de AlN depositadas en este trabajo aseguran un comportamiento estable en cuanto a estructura y a propiedades mecánicas para temperaturas de hasta $850^{\circ} \mathrm{C}$. Además, las técnicas de nanoindentación y pin-on-disk coinciden en mostrar la capa sometida a tratamiento térmico a $850^{\circ} \mathrm{C}$ como la que ofrece mayor dureza y resistencia al desgaste, superando así las propiedades del nitruro de aluminio original. Por esta razón, el nitruro de aluminio se plantea como un material candidato a ser utilizado como recubrimiento protector en procesos industriales, especialmente los que tengan lugar a temperaturas en torno a $850^{\circ} \mathrm{C}$.

TABLA I. VALORES DE DUREZA DE LOS RECUBRIMIENTOS DE TIN Y ALN ANTES Y DESPUÉS DE LOS TRATAMIENTOS TÉRMICOS.

\begin{tabular}{|l|l|l|}
\hline & Dureza TiN & Dureza AlN \\
\hline Original & $23.92 \pm 1.07$ & $16.51 \pm 0.16$ \\
\hline $\mathrm{T}=750^{\circ} \mathrm{C}$ & $10.90 \pm 2.70$ & $15.72 \pm 0.21$ \\
\hline $\mathrm{T}=850^{\circ} \mathrm{C}$ & $6.19 \pm 1.67$ & $16.91 \pm 0.70$ \\
\hline $\mathrm{T}=950^{\circ} \mathrm{C}$ & $3.82 \pm 1.74$ & $1.65 \pm 0.64$ \\
\hline
\end{tabular}




\section{CONCLUSIONES}

En este trabajo se han depositado capas de TiN y AlN mediante la técnica de sputtering magnetrón reactivo. Se ha analizado su estructura cristalina, morfología superficial, dureza y resistencia al desgaste antes y después de realizar tratamientos térmicos a diferentes temperaturas $(750,850 \mathrm{y}$ $\left.950^{\circ} \mathrm{C}\right)$, y se han obtenido las temperaturas de estabilidad para ambos materiales.

Las capas de TiN conservan su estructura cristalina hasta una temperatura de $750^{\circ} \mathrm{C}$, la cual se confirma con los resultados obtenidos para su resistencia al desgaste.

La estabilidad térmica de las capas de AlN se sitúa en torno a $850^{\circ}$, aunque en este material se observa una mejoría en la respuesta estructural y mecánica al aplicar temperatura. Con un tratamiento térmico a $850^{\circ} \mathrm{C}$ se obtienen mejores propiedades que las del recubrimiento original, ya que se consigue un valor más alto de dureza que la ofrecida por el nitruro de aluminio a temperatura ambiente.

Se verifica así la utilización de capas de TiN o AlN como recubrimientos protectores en procesos industriales a alta temperatura.

\section{AGRADECIMIENTOS}

Los autores agradecen la financiación recibida por los proyectos MAT 2002-04037-C03-03 del Ministerio de Educación y Ciencia y GR/MAT / 0716/ 2004 de la Comunidad de Madrid, gracias a la cual ha sido posible la realización del presente trabajo.

\section{BIBLIOGRAFÍA}

1. L. Cunha, M. Andritschky, K. Pischow, Z. Wang, A. Zarychta, A.S. Miranda, A.M. Cunha «Performance of chromium nitride and titanium nitride coatings during plastic injection moulding» Surf. Coat. Technol. 153 [2-3] 160-165 (2002).

2. J.F. Marco, A.C. Agudelo, J.R. Gancedo, D. Hanzel. « Surface spectroscopic study of the behaviour of a thin TiN layer as protective coating of iron against corrosion by humid SO2-aggressive environments» Surf. Interface Anal. 26 [9] 667-673 (1998).

3. J. Hillman, R. Foster, J. Faguet, W. Triggs, R. Arora, M. Ameen, F. Martin, C. Arena. «Integrated CVD titanium and titanium nitride processes for sub0.5-mu-m metallization» Solid State Technol. 38 [7] 147-\& (1995)

4. A. Cantarero, C. Ferrer, A.S. Bolinches, B. Straumal, N. Vershinin, E. Rabkin. «Vacuum arc deposition of $\mathrm{Ti}, \mathrm{TiN}$ and $\mathrm{TiO} 2$ decorative coatings on glass» Bol. Soc. Esp. Cerám. V. 40 [2] 138-142 (2001).

5. M.A. Auger, O. Sánchez, J.M. Albella. «TiN deposition on cutting tools for industrial use» Bol. Soc. Esp. Cerám. V. 43 [2] 488-490 (2004)

6. V. Mortet, A. Vasin, P.Y. Jouan, O. Elmazria, M.A. Djouadi. «Aluminium nitride films deposition by reactive triode sputtering for surface acoustic wave device applications» Surf. Coat. Technol 176 [1] 88-92 (2003)

7. S.P. McGeoch, F. Placido, Z. Gou, C.J.H. Wort, J.A. Savage. «Coatings for the protection of diamond in high-temperature environments» Diam. Relat. Mater. 8 [2-5] 916-919 (1999).

8. M.A. Auger, R. Gago, M. Fernández, O. Sánchez, J.M. Albella «Deposition of TiN/AlN bilayers on a rotating substrate by reactive sputtering» Surf. Coat. Technol. 157 [1] 26-33 (2002).

9. W.C. Oliver, G.M. Pharr. «An improved technique for determining hardness and elastic-modulus using load and displacement sensing indentation experiments» J. Mater. Res. 7 (6) 1564-1583 (1992).

10. PCPDFWIN versión 2.2 (C) 2001 JCPDS-ICDD.

11. M. Wittling, A. Bendavid, P.J. Martin, M.V. Swain. «Influence of thickness and substrate on the hardness and deformation of TiN films» Thin Solid Films 270 [1-2] 283-288 (1995).

Recibido: 14.01.05

Aceptado: 23.12.05 